\title{
PROPUESTAS PARA AFRONTAR LOS RETOS DEL EUROPEAN CREDIT TRANSFER SYSTEM (ECTS) EN ASIGNATURAS UNIVERSITARIAS DE REDACCIÓN PERIODÍSTICA ${ }^{1}$
}

\author{
Carles Pont Sorribes \\ carles.pont@upf.edu \\ Sergi Cortiñas Rovira \\ zsergi.cortinas@upf.edu \\ (Universitat Pompeu Fabra)
}

Resumen:

Los retos del nuevo paradigma comunicativo, un cruce histórico lleno de posibilidades pero también de riesgos y límites, obligan a una redefinición en la educación superior de los futuros profesionales de la comunicación. Este trabajo, realizado a partir de las experiencias de dos profesores universitarios, propone la introducción del hipertexto en la docencia universitaria en el campo del periodismo y analiza las ventajas que esta nueva herramienta supone en la formación de los periodistas del siglo XXI. En esta investigación los autores proponen el hipertexto como metodología de trabajo en el aula, suponiendo que el hipertexto tiene que ser concebido, como se verá en esta investigación, como el locus en el que deben converger la teoría y la práctica, donde tiene que explotarse la capacidad crítica y la creatividad del alumno y en el que debe potenciarse la interactividad profesor-alumno y alumno-alumno.

Palabras clave: Educación en periodismo, hipertexto, redacción periodística, nuevas tecnologías, comunicación audiovisual.

\section{Abstract:}

The challenges of the new communicative paradigm, a historical crossroads full of possibilities but also of risks and limits, force to a redesign of learning in communication. This work, carried out from the experiences of two university teachers, proposes the introduction of the hypertext in the university teaching of journalism and analyzes the advantages that this new tool supposes in the education of the media professionals of the 21 st century.

Keywords: Journalism education, hypertext, writing news, new technologies, audiovisual communication.

\footnotetext{
${ }^{1}$ Este trabajo forma parte del ámbito del proyecto de investigación "La competencia receptiva crítica en estudiantes universitarios y de bachillerato: análisis y propuesta didáctica" (HUM2004-03772/FILO; 20052007), financiado por la Dirección General de Enseñanza Superior e Investigación Científica, cuyo investigador principal es el doctor Daniel Cassany (UPF).
} 


\section{INTRODUCCIÓN}

a sociedad de la información pone al servicio de los profesionales de la docencia universitaria una gran cantidad de herramientas. Pocas veces antes en la historia de la humanidad habíamos asistido a una miríada de novedades tecnológicas como las que tenemos actualmente. Este hecho obliga a un replanteamiento de la docencia desde todos los ámbitos.

La comunicación, dentro y fuera del aula, tiene que incentivarse, tanto en el sentido profesor-alumno como alumno-alumno. Se trata, pues, de potenciar las herramientas que metodológicamente permitan fomentar la interactividad entre ambos colectivos y hacer crecer, al mismo tiempo, la practicidad de las asignaturas. En este sentido, este artículo propone un método pedagógicamente inductivo con ejercicios que los mismos alumnos puedan realizar desde un hipertexto, que en el caso de la Universitat Pompeu Fabra puede asimilarse al Aula Global .

De una manera indirecta, la propuesta pone sobre la mesa la necesidad de una mejora de las habilidades tecnológicas de los alumnos, teniendo en cuenta que los nuevos tiempos favorecen una mayor destreza de los estudiantes. Así, una vez erradicado el analfabetismo clásico, la sociedad trata de eliminar el analfabetismo tecnológico, la epidemia educativa más importante de nuestros tiempos. Éste es uno de los grandes retos de futuro y exigirá una constante formación también por parte de los docentes. Es por este motivo que la iniciativa que aquí se plantea tiene una doble vertiente que permite mejorar, a la vez, dos puntos fundamentales de la docencia: la simplificación de la clase magistral y la introducción de las nuevas tecnologías en la docencia universitaria, en línea con aquello que propugna el Espacio Europeo de Educación Superior (EEES), también conocido como Horizonte Bolonia, en lengua inglesa llamado European Credit Transfer System (ECTS).

Aún así, el riesgo de menospreciar la formación teórica sólida se divisa como una consecuencia directa de una educación excesivamente práctica y tecnológica. Se trata, en esencia, de un falso dilema, puesto que los conocimientos teóricos deben ser paralelos y compatibles con la practicidad de los estudios y el fomento de las habilidades tecnológicas de los alumnos.

\section{OBJETIVOS Y METODOLOGÍA DE LA INVESTIGACIÓN}

Los objetivos de este artículo se encaminan a la mejora de la docencia universitaria en la redacción periodística audiovisual a través de las nuevas tecnologías. Estos objetivos se pueden dividir en tres puntos:

1. Proponer un método, basado en el hipertexto, que potencie las habilidades tecnológicas a través del aprendizaje inductivo de las asignaturas de redacción periodística en estudios universitarios.

\footnotetext{
2 Aula Global es el nombre que en la UPF recibe el entorno web en el que los alumnos y los profesores pueden interactuar.
} 
2. Incentivar la interactividad entre el profesor y el alumno a partir de las herramientas que facilita el hipertexto, y permitir la interrelación entre los mismos alumnos para potenciar el aprendizaje significativo.

3. Mejorar la practicidad de las asignaturas para la formación de periodistas y comunicadores, mediante la realización de ejercicios prácticos relacionados con la actualidad.

La metodología de esta investigación se basa en la técnica de la observación participante u observación de campo, especialmente útil para el investigador para definir la información de los antecedentes básicos necesarios para formular hipótesis y aislar las variables dependientes e independientes (Wimmer y Dominick, 2001: 112). Este tipo de investigación, que lleva a cabo la observación real del objeto investigado in situ, ha sido muy prolífica en estudios académicos como la investigación sobre el funcionamiento de los informativos en cadenas de televisión de Epstein (1974), o los trabajos sobre rutinas profesionales de los informadores, entre muchos otros.

Para desarrollar la técnica de la observación participante los dos profesores de la asignatura se han intercambiado los roles, siendo investigadores y profesores en tiempos diferentes. Así, en un primer estadio, el profesor ' $A$ ' ha sido observado por el profesor 'B', éste en el rol de investigador y, en un segundo proceso, el profesor ' $A$ ', haciendo de investigador, ha observado al profesor ' $B$ ', ahora en el papel de docente.

El método de la observación participante entrecruzada por los dos investigadores ha permitido que no se mezclaran los papeles de investigador y profesor en un mismo momento del proceso y, a la vez, ha sido útil para extraer resultados más objetivos. A través de esta técnica, se ha podido describir y explicar con detalle la experiencia docente de los dos autores de esta investigación en la asignatura Técnicas de Redacción Periodística, de los Estudios de Comunicación Audiovisual de la Universitat Pompeu Fabra de Barcelona ${ }^{3}$.

La metodología de trabajo también se ha completado con el análisis de las encuestas que responden los alumnos cuando acaban el curso. La Universitat Pompeu Fabra de Barcelona pone a disposición de los discentes las encuestas para evaluar la docencia recibida por parte de los profesores (Encuestas de Evaluación Docente, EAD). Éstas tienen un apartado cuantitativo donde el alumno valora del 1 al 10 aspectos como la puntualidad, la idoneidad de los materiales docentes o la claridad en las explicaciones del profesor, entre otros. Las encuestas también disponen de una parte cualitativa en la que puede anotar reflexiones sobre la asignatura. Es este apartado en el que nos hemos fijado especialmente para elaborar esta investigación. Se han tomado como referencia los resultados cualitativos de los últimos cuatro cursos

\footnotetext{
3 Sergi Cortiñas imparte dos grupos de esta asignatura ininterrumpidamente desde el curso 2000-2001, hasta la actualidad. Carles Pont imparte dos más desde el curso 2001-2002 hasta la actualidad (curso 2006-2007). La asignatura, que se desarrolla en el segundo curso (de cuatro en total), tiene tres créditos y es una materia troncal en el plan de estudios. Técnicas de Redacción Periodística es una materia que pertenece a la cátedra de Periodística de la UPF, que dirige el catedrático Josep Maria Casasús. El área de Periodística de los Estudios de Periodismo está coordinada por el doctor Carles Singla.
} 
académicos, desde el año 2003 hasta el 2007, coincidiendo con la introducción del nuevo método que aquí se desarrolla.

Finalmente, como fuentes primarias de la metodología, se ha realizado un vaciado sistemático de bibliografía docente relacionada con el Espacio Europeo de Educación Superior (Horizonte Bolonia), así como otros materiales procedentes de áreas de conocimiento como la Periodística, la Teoría del Periodismo, la Teoría de la Comunicación y la Documentación.

\section{MARCO TEÓRICO: ALGUNAS CONSIDERACIONES DEL CONCEPTO HI- PERTEXTO}

La definición de hipertexto ha sido objeto de constantes reinterpretaciones desde que Vannevar Bush usó el concepto por primera vez en el memorable texto As we may Think (1945). El término hipertexto ha venido designando, a lo largo del tiempo, tanto una forma concreta de estructurar la información, como un ideal, muy abstracto, referido a cómo organizar la totalidad del conocimiento humano, y de aquí la dificultad para definirlo y las confusiones que genera con frecuencia el concepto (Codina, 1997-1998).

Técnicamente, un hipertexto es un conjunto de nudos atados por conexiones. Los nudos pueden ser palabras, imágenes, grafismos, secuencias audiovisuales o documentos enteros que también pueden ser de otros hipertextos (Lévy, 1990 y 1991). Los ítems no están conectados linealmente, como los nudos de una cuerda, sino en forma de estrella, según un modelo reticular. Navegar por un hipertexto puede suponer diseñar un recorrido por una red tan compleja como se quiera (Tomàs, 1997). Según George Landow (1992), el hipertexto puede asimilarse a un medio informático que relaciona información, tanto verbal como no verbal. Los nexos electrónicos unen lexias (según las teorías defendidas por Barthes, fragmentos de texto) externas y/o internas a la obra (textos de apoyo de otros autores, por ejemplo), creando un texto que el lector experimenta como no lineal o, mejor dicho, como multilineal o multisecuencial (Tomàs, 1997). Desde este punto de vista, es oportuna la doble definición de Codina (1997-1998), que a efectos del presente trabajo resulta sumamente aclaratoria: a) un hipertexto es una estructura de la información que organiza un conjunto de elementos en forma de red, y b) un conjunto de elementos está organizado en forma de red cuando existe alguna forma de unión entre ellos, pero no existe un orden único de recorrido entre ellos.

Un hipertexto, según el modelo que quedó establecido en los años 80 y que fue consagrado posteriormente por el lenguaje HTML (Canals, 1990; Nielsen, 1995; Woodhead, 1991; Caridad y Moscoso, 1991; Díaz, Catenazzi, Aedo, 1996; Tittel et al., 1996), consiste en tres elementos, a saber: a) un conjunto de nodos, que son los elementos que contienen la información, b) los enlaces entre estos nodos, y c) el conjunto de anclajes que identifican el inicio y el destino de cada enlace, o qué conecta con qué (Codina, 1997-1998). 


\subsection{Hipertexto y docencia en la redacción periodística audiovisual}

En este artículo, se propone que el profesional de la docencia aproveche, cada cual en función de los intereses particulares de cada materia, las ventajas del hipertexto, como herramienta de apoyo básica para el desarrollo satisfactorio de los objetivos del curso de redacción periodística. Este hipertexto tendría que convertirse en un gran locus docente en el cual convivan herramientas teóricas, herramientas prácticas e información textual, gráfica, sonora y visual.

En este hipertexto el alumno debe poder adquirir los conocimientos teóricos básicos. Éstos los podrá confrontar con ejemplos de textos, imágenes y documentos sonoros de radios y televisiones profesionales con un acceso directo, que el profesor relacionará previamente con el contenido teórico de la asignatura.

El estudiante absorbe el conocimiento teórico con ejemplos reales, hecho que le permite comprobar errores y aciertos de la redacción audiovisual y los relaciona con la práctica profesional de los medios de comunicación. Una vez asumida la teoría por la vía de los ejemplos, el alumno podrá practicar con casos reales cuando hace los ejercicios, que generalmente son escribir una noticia o un reportaje para un medio audiovisual (alternativamente radio y televisión).

En la parte práctica, el estudiante tiene que identificar y seleccionar las fuentes de la información en Internet (textos de agencia de noticias, bases de datos online, cortes de voz, archivos sonoros, bancos de imágenes), editar digitalmente las piezas informativas, locutarlas en calidad digital y escuchar su propia locución. En estas condiciones, se favorece que el talento y la creatividad del alumno se manifiesten plenamente, pero a la vez se permite que sea el alumno quien logre los objetivos de la asignatura a través de sus errores y aciertos (método inductivo).

La organización docente de la materia responde a los principios básicos que plantea el Espacio Europeo de Educación Superior con un contenido equilibrado entre sesiones magistrales, sesiones prácticas y de seminario y la tutorización individual (PQE, 2006: 26). La asignatura se organiza en función de las sesiones programadas (sistema Syllabus anglosajón), un total de diez sesiones de tres horas cada una. El curso se inicia con dos/tres clases en las cuales se explica toda la parte teórica de la redacción para medios audiovisuales. Este primer estadio, si bien tiene su parte magistral, se desarrolla también en formato hipertextual. Por ejemplo, cuando se explica el uso del complemento circunstancial de lugar en las noticias audiovisuales, los alumnos tendrán la oportunidad de escuchar diferentes piezas profesionales de emisoras de radio o televisión que las utilizan adecuadamente y otras que no lo hacen. El ejercicio aquí propuesto permite que el discente pueda relacionar mejor la teoría que se imparte con la parte práctica del curso.

Tras las sesiones teóricas, la siguiente clase sirve para saber si se han adquirido estos conocimientos a través de un cuestionario individual (examen en términos clásicos). A partir de este punto, después de elaborar el cuestionario, se inicia la parte más práctica de la asignatura en el que cada alumno deberá seguir los cuatro pasos que se desarrollarán más adelante en esta investigación: a) recopilación de información y background, b) producción de la noticia, c) locución de la noticia y publicación de materiales, d) evaluación mancomunada y tutorización individual. 
Por lo tanto, y en resumen, en cada sesión el alumno tiene que elaborar todo el proceso productivo de la noticia, desde la recopilación de datos hasta la locución final. La corrección por parte del docente se hace siempre entre una sesión y la siguiente. El estudiante conoce su evolución una vez el profesor ha hecho una corrección definitiva y siempre después de haber puesto en común en clase con los compañeros el método de la evaluación mancomunada que se compara con las aportaciones de las piezas radiofónicas y/o televisivas profesionales de los distintos medios de comunicación de masas.

\section{RESULTADOS}

Mediante la técnica de la observación participante se ha llegado a los resultados que se muestran a continuación. Para describir este Aula hipertextual (también citado en este artículo como hipertexto docente, interfaz hipertextual, o simplemente hipertexto) en la asignatura Técnicas de Redacción Periodística para medios audiovisuales se han distinguido cuatro fases muy diferenciadas y fundamentadas en el método pedagógico inductivo, que los discentes deben seguir durante el curso:

\subsection{Recopilación de información y background}

Para empezar, es necesario que los alumnos accedan al hipertexto de la asignatura en el cual encontrarán las fuentes de donde han de extraer la información básica para redactar las noticias. En esta ventana, optarán a redireccionamientos hacia las páginas de las principales agencias de noticias del mundo. En la medida que sea posible, y de acuerdo con los convenios que tenga la universidad en cuestión, podrán localizar unos contenidos u otros. Las informaciones (teletipos de agencia de noticias) deben estar relacionados siempre con temas actuales o siguiendo las directrices que marque el docente en cada caso.

Por otra parte, el profesor de la asignatura debe confeccionar una parrilla de gabinetes de prensa que mediante links deben ser consultables; es interesante que el alumno pueda escoger entre una gran variedad. En este sentido, el profesor selecciona los mejores sitos web que contienen notas de prensa e información general de diferentes sectores ${ }^{4}$. Los datos que obtengan los alumnos de la página hipertextual podrán complementarse con recursos propios, como, por ejemplo, entrevistas con expertos relacionados con la temática que se quiere tratar, búsqueda de fuentes complementarias, portafolios, documentación bibliográfica, etc., entre otras alternativas.

Una vez que el estudiante haya hecho la inmersión necesaria en el tema de la noticia, el profesor revisará la propuesta y sugerirá posibles cambios o soluciones al planteamiento. El docente hará recomendaciones en función de aquellos conocimientos teóricos que se han adquirido y concluirá sobre la oportunidad, o no, de las distintas iniciativas que puedan suscitarse.

\footnotetext{
4 Por ejemplo, se puede escoger del ámbito económico empresas de gas, agua, bancos, eléctricas, energía, etc., del ámbito político, administraciones, partidos, asociaciones, ONG's, etc., del ámbito cultural, compañías teatrales, productoras de cine, instituciones, etc., del ámbito deportivo, clubes, deportistas, federaciones...
} 


\subsection{Producción de la noticia}

Cuando el discente ha recogido toda la información elabora su noticia a partir de unos criterios preestablecidos por el docente que responden, sobre todo, a un modelo de formato y un método estandarizado (género periodístico, estilo, estructura informativa, tono de la pieza) para todos los estudiantes. En esta fase el alumno también debe recoger informaciones sonoras que editará para convertirlas en lo que en periodismo audiovisual se denominan los cortes de voz en radio o inserts en televisión.

Tras escoger la información sonora y redactar el texto, el alumno recibe la supervisión de su trabajo por parte del docente. En este estadio, el profesor hace indicaciones de tipo metodológico, pero sin corregir a fondo el contenido, trabajo que se compartirá con el alumno al final del proceso. Ésta es una fórmula mixta entre la autoevaluación y la corrección del profesor, que en el caso descrito ha dado resultados satisfactorios.

\subsection{Locución de la noticia y publicación de materiales}

Uno de los mejores métodos para comprobar si aquello que se ha escrito responde a las exigencias mínimas que debe tener un texto para ser leído es locutarlo y hacer una grabación. Con este objetivo, el alumno se desplaza al estudio de radio o cabina de edición de televisión, donde, con el texto previamente redactado y con los cortes de voz o inserts escogidos, confecciona la noticia en formato sonoro.

En esta etapa del proceso los alumnos reciben la ayuda de un técnico de sonido especializado que los apoya en aspectos prácticos y técnicos. Una vez grabada la noticia, el estudiante escucha la noticia que ha leído y la compara con la parte teórica de la asignatura que puede encontrar en el hipertexto.

Este ejercicio es una autoevaluación previa que se deberá cotejar con la que al final del proceso hará el profesor. Al acabar, los alumnos publican su noticia en formato sonoro y escrito en el hipertexto para que todos los estudiantes puedan leer y evaluarse mancomunadamente. La publicación del trabajo es esencial para el pleno desarrollo del proceso de aprendizaje, puesto que ayuda a dar visibilidad a su texto y hacerlo susceptible de las aportaciones de los compañeros. El alumno aprende tanto de aquello que él hace bien o mal, como de lo que ve que sus compañeros hacen.

\subsection{Evaluación mancomunada y tutorización individual}

El profesor dedica un tiempo -en el caso analizado, una media hora aproximadamente- al final de cada sesión a la evaluación mancomunada. En estos minutos los protagonistas son los alumnos que intervienen para comentar los textos propios y los de los compañeros, al objeto de aportar soluciones o mejoras a las piezas radiofónicas/televisivas elaboradas por ellos mismos o sus compañeros.

En paralelo, el profesor busca la comparación de las piezas elaboradas por los alumnos con las teorías de la cuestión y, puntualmente, con determinadas informaciones profesionales que también encontrará en la interfaz hipertextual que el pro- 
fesor le propone para abordar el curso. Este método permite cotejar aquellas piezas que están confeccionadas a partir del modelo propuesto en la asignatura y otras que se pueden usar en el ámbito profesional. Así se fomenta que los estudiantes adquieran habilidades para discernir entre diferentes modelos de redacción y los comparen con aquellos formatos que más se adaptan a un modelo óptimo. Al mismo tiempo, se quiere conseguir que el alumno tenga una actitud crítica respeto a los productos que se elaboran en clase, pero también respecto a los productos profesionales actuales. Este espíritu crítico es una de las bases del aprendizaje activo y significativo que propone el EEES y ayuda a dotar al alumno de un protagonismo que en las lecciones magistrales clásicas apenas tenía.

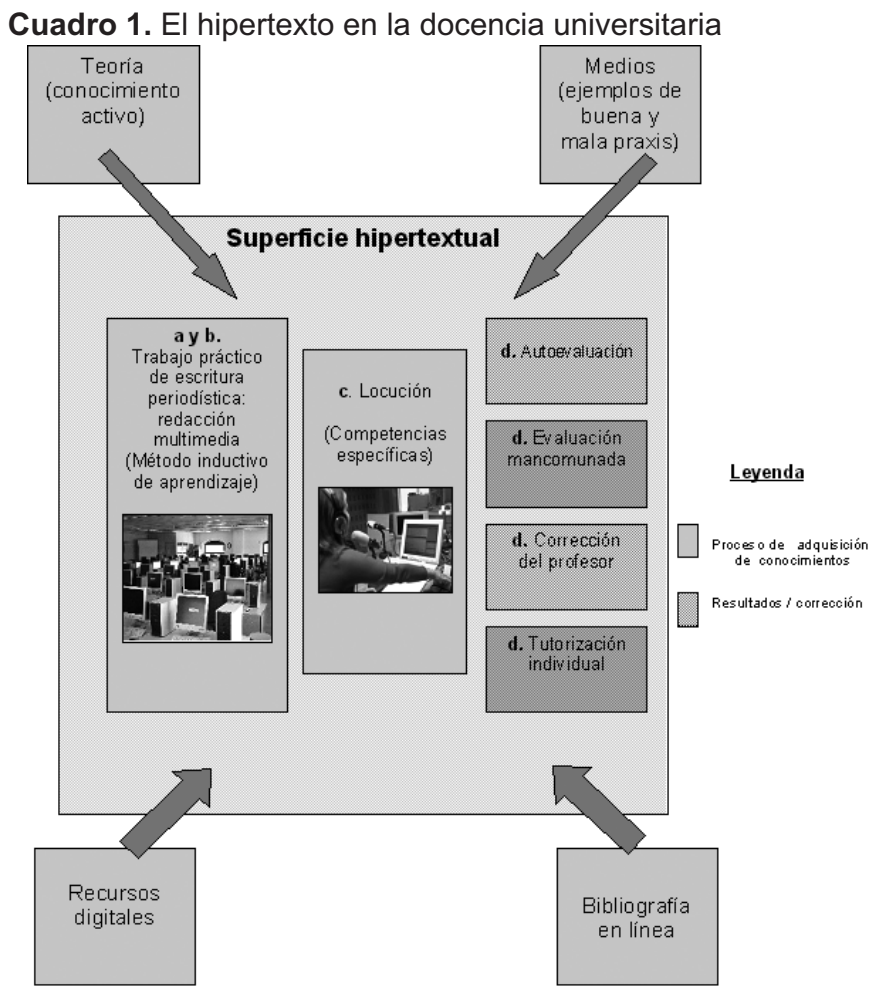

Fuente: elaboración propia.

En este sentido, el presente método fundamenta las competencias transversales, que son todos aquellos conocimientos que tiene que aprender un estudiante más allá de los requisitos específicos de una materia", como por ejemplo el trabajo en equipo, la expresión oral, la expresión escrita u otras, como en este caso la capacidad crítica (PQE-UPF, 2006: 18). El profesor, mientras tanto, se convierte en una especie de gatekeeper ${ }^{5}$ docente que filtra y controla los contenidos que elabora el alumno.

El estudiante puede ir viendo su evolución a lo largo de las diferentes sesiones que se desarrollan, puede escuchar sus piezas grabadas al inicio del curso, rehacerlas, observar los aciertos o anotar los errores para enmendarlos. Al mismo tiempo, el es- 
tudiante podrá comparar su trabajo con el de sus compañeros de clase, ejercicio que, desde el nuestro punto de vista, le motiva a participar en la propia dinámica de grupo.

El proceso finaliza con la tutorización individual profesor-alumno. En este punto, el alumno tendrá nuevamente una valoración por parte del docente de un modo más privado. Así el estudiante podrá relacionar las autocorrecciones con la sesión mancomunada y con las indicaciones que, ahora sí, le hará el profesor en su texto. Durante la clase, el profesor se reúne en una sesión individual (tutorización individual, según el EEES) con cada alumno y revisa los contenidos de los textos, explica las correcciones y las consensúa con sus puntos de vista. El alumno obtiene la nota final teniendo en cuenta una evaluación continua durante todo proceso.

La revisión mancomunada y la tutorización individual pueden conducir, de nuevo, a la publicación de las piezas en la página hipertextual, hecho que permite volver al inicio del proceso. Este proceso iterativo realizado en una situación ideal a lo largo de $n$ veces daría como resultado una pieza formalmente perfecta.

Por otro lado, los alumnos también evalúan al profesor al final del proceso de aprendizaje, siempre antes de que los discentes conozcan la calificación definitiva del curso para que ésta no influya en sus opiniones. Esta evaluación puede ser consultada por el profesor y los responsables académicos del Decanato, del Departamento y de la Universidad. En estos formularios, los alumnos responden a una serie de preguntas sobre la docencia del profesor de la asignatura mediante la intranet de la Universidad de modo totalmente confidencial, con el aval institucional y bajo la supervisión del Vicerrectorado de Programación, Docencia y Evaluación. Para esta investigación se han considerado los cursos 2003-2004, 2004-2005, 2005-2006 y 2006-2007. Entre los resultados destaca que los alumnos consideran el nuevo método provechoso y original, además, de calificar de bien planteada la forma de evaluación. En cambio, los discentes opinan, como punto débil del sistema docente, que el ritmo seguido en clase es, en ocasiones, demasiado estresante, algo que de algún modo está en consonancia con una de las características intrínsecas de la profesión periodística.

\section{CONCLUSIONES}

La introducción del hipertexto, como herramienta docente, optimiza el aprendizaje significativo ${ }^{6}$ del alumno en las clases de redacción periodística.

Este artículo propone soluciones eminentemente prácticas para la introducción de las nuevas tecnologías en las aulas convencionales en asignaturas de Redacción Periodística audiovisual. La introducción del método del hipertexto nos ha per-

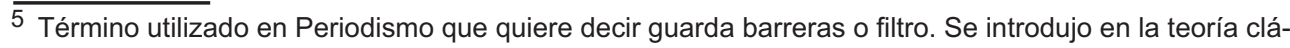
sica del Periodismo a partir del estudio de David Manning White, The Gatekeeper: A Case Study in the Selection of News.

6 El término aprendizaje significativo forma parte de la teoría del mismo nombre del pedagogo David Paul Ausubel y se define como aquel aprendizaje en el cual el alumno elabora e internaliza los conocimientos a partir de experiencias anteriores. Se trata de un aprendizaje considerado con sentido, para diferenciarlo de aquellos aprendizajes puramente mecánicos o memorísticos.
} 
mitido comprobar que se ha mejorado uno de los objetivos básicos de la asignatura, esto es, el aprendizaje de las técnicas de redacción de textos tanto para radio como para televisión. La evolución del aprendizaje ha sido significativo cuando los alumnos han podido grabar sus textos y después escucharlos y revisarlos conjuntamente con el profesor. De esta manera, se ha conseguido que el alumno detecte los errores que comete y visualice rápidamente los puntos en los cuales progresa y en los que tiene más problemas de asimilación.

Las nuevas tecnologías aplicadas en esta investigación mejoran la motivación de los discentes y el aprendizaje de tipo activo, de acuerdo con los objetivos del European Credit Transfer System (ECTS).

Con este método se consigue que haya una motivación constante en todo el proceso de aprendizaje y sirve de estímulo para llevar a cabo actividades compartidas tanto dentro como fuera del aula (entrevistas, reportajes, recopilación de información, etc.). A través del sistema anteriormente explicado, se potencia una enseñanza de tipo activo, en línea con las estrategias docentes del European Credit Transfer System (ECTS). Al mismo tiempo, se constata a lo largo de la experiencia docente que el alumno muestra una satisfacción creciente al comprobar que sus expectativas se cumplen, si se tienen en cuenta las opiniones reflejadas en las evaluaciones de los estudiantes.

La nueva propuesta docente procura el logro de las competencias denominadas específicas y éstas, a su vez, contribuyen a la adquisición de otras competencias transversales, tales como el espíritu crítico, la creatividad y la reflexión.

A partir del método de trabajo expuesto en este artículo se fomenta uno de los aspectos esenciales de las nuevas directrices del paradigma docente que supone el nuevo marco del Espacio Europeo de Educación Superior: las competencias. La propuesta que se presenta en esta investigación profundiza en el logro de las competencias denominadas específicas, tal y como se recoge en los propósitos del EEES, y a la vez, otras competencias transversales (reflexión sobre cómo trabajan los medios, el espíritu crítico profesional, la expresión oral y escrita, la creatividad. etc.). En especial, el método pretende hacer desarrollar la capacidad crítica de los alumnos, uno de los retos de la enseñanza actual (Cassany, 2003; Borrat, 2005; Kanpol, 1994; Giroux, 1988). Existe un amplio consenso en que el discente no sólo debe saber redactar de manera satisfactoria sino que debe ser capaz de reflexionar sobre cómo actúan los medios de comunicación y los grupos empresariales que hay detrás, así como saber discernir los intereses que les rodean. En buena medida, esto se puede conseguir a partir de un método de trabajo como el que aquí se expone, que obliga al alumno a un proceso continuo de toma de decisiones, que fomenta el razonamiento en cada paso y le obliga a dotarse de un criterio de trabajo lógico, coherente y crítico. 


\section{REFERENCIAS BIBLIOGRÁFICAS}

BORRAT, H. Periódicos de calidad: primeras propuestas para una lectura crítica. Portal de la Comunicació. Institut de la Comunicació UAB. [en línea] 2005. [Consulta: 1 junio 2006]. <www.portalcomunicacion.com/esp/pdf/aab_lec/21.pdf>

- (2003) Las relaciones noticiables fuentes-autores. En: LOSADA, A. y ESTEVE RAMÍREZ, F. El periodismo de fuente. Salamanca: Publicaciones de la Universidad de Salamanca, p. 67-84.

CASSANY, D. La cuina de l'escriptura. Barcelona: Les Naus d'Empúries, 1993.

- (2003) "Aproximaciones a la lectura crítica: teoría, ejemplos y reflexiones". Tarbiya. Revista de investigación e innovación educativa, núm. 32, (2003) p.113-132.

CASASÚS, J. M. Iniciación a la Periodística. Barcelona: Teide, 1988.

CODINA, L. "H de Hypertext, o la teoría de los hipertextos revisitada", Cuadernos de documentación multimedia, núm. 6-7 (1997-1998).

DÍAZ NOCI, J.; MESO AYERDI, K. Periodismo en Internet. Bilbao: Servicio de Publicaciones de la Universidad del País Vasco, 1999.

DIJK, T. Van. La noticia como discurso: comprensión, estructura y producción de la información. Barcelona: Paidós, 1990.

GIROUX, H. A. Teachers as Intelectuals. Towards a Critical Pedagogy of Learning. Massachusetts: Bergin \& Garvey, 1988.

GOMIS L. Teoria dels gèneres periodístics. Barcelona: Centre d'Investigació de la Comunicació, 1989.

GRIJELMO, Á. El estilo del periodista. Madrid: Taurus, 1997.

IMBERNON MUÑOZ, F.; MEDINA MOYA, J. L. "Metodologia participativa a l'aula universitària. La participació de l'alumnat". Collecció Quaderns de Docència Universitària, núm. 4 (2005).

JENSEN, K. B,; JANKOWSKI, N. W. (eds.) Metodologías cualitativas de investigación en comunicación de masas. Barcelona: Bosch, 1993.

KANPOL, B. Critical Pedagogy. An Introduction. Londres: Bergin \& Garvey, 1994.

LANDOW, G. Hypertext: The Convergence of Contemporary Critical Theory andtechnology. Baltimore: John Hopkins Univ. Press, 1992.

LÉVY, P. L'idéographie dynamique. París: La Découverte, 1991.

- (1990) Les Technologies de l'intelligence. París: La Découverte.

MACDOUGAL, C. D. Interpretative reporting. Nueva York: Macmillan, 1987.

PROGRAMA PER LA QUALITAT EDUCATIVA (PQE). Manual d'introducció a la- 
docència: Horitzó Bolonya. Barcelona: Universitat Pompeu Fabra, 2006.

RAMONET, I. El periodismo del nuevo siglo . La Factoria [en línea]. Febrero de 1999, $\mathrm{n}^{\circ}$ 8. [Consulta: 3 mayo 2006]. <http://www.lafactoriaweb.com/articulos/ramonet.htm>

REIG, R. La comunicación en su contexto: una visión crítica desde el periodismo. Sevilla: Centro Andaluz del Libro, 2002.

RESOLDRE PROBLEMES PER APRENDRE. Segundo volumen. Colección EINES, Unitat d'Innovació Docent en Educació Superior (IDES). Barcelona: UAB, 2005.

SANS, A. "L'avaluació dels aprenentatges: construcció d'instruments". Colección Quaderns de Docència Universitària, núm. 2, ICE-UB, (2004).

SITJÀ, X Y OLIVA, L. Les notícies a la televisió. Barcelona: RTVE, 1990.

TOMÀS, C. "L'hipertext i les obres obertes. Una aproximació des de la teoria cultural". Documento elaborado para la asignatura el medio interactivo, dirigida por el doctor Xavier Berenguer, que forma parte del doctorado en Comunicación Audiovisual de la Universitat Pompeu Fabra y del Instituto Universitari de l'Audiovisual, BarceIona, 1997. [Consulta: 1 junio 2006]. <http://www.iua.upf.es/ ctomas/ctp47.htm>

TÚÑEZ, M. Producir noticias. Cómo se fabrica la realidad periodística. Santiago de Compostela: La Verde Ediciones, 1999.

TUCHMAN, G. La producción de la noticia. Estudio sobre la construcción de la realidad. Barcelona: Gustavo Gili, 1983. p. 55-66-67.

WIMMER, R. y DOMINICK, J. Introducción a la investigación de medios masivos de comunicación. Mexico D.F: Internacional Thomson Editores, 2001.

\section{Breve semblanza de los autores}

Carles Pont Sorribes es profesor de Periodismo y Comunicación Audiovisual en la Universitat Pompeu Fabra, e imparte las asignaturas Técnicas de Redacción Periodística, Comunicación y Opinión Pública y Seminario de Análisis de la Actualidad. Es autor de la Tesina "Periodismo de Crisis: aproximación Teórica y Tipológica" y ha participado en investigaciones sobre campañas electorales y comunicación, análisis de la prensa en Andorra y medios de comunicación en situación de crisis.

Sergi Cortiñas Rovira se licenció en Ciencias Químicas por la Universitat de Barcelona y en Periodismo por la Universidad Pompeu Fabra. Desde el año 1999 es profesor e investigador en el Departamento de Periodismo y de Comunicación Audiovisual. Es doctor en Comunicación Social desde septiembre de 2006, con una tesis sobre periodismo científico. 